\title{
O PROGRAMA DE TRATAMENTO FORA DE DOMICÍLIO NO SISTEMA ÚNICO DE SAÚDE NO PIAUÍ
}

\author{
S. R. AZEVÊDO \\ Universidade Federal do Piauí - UFPI \\ azevedosandro@ig.com.br
}

Artigo submetido em fevereiro/2016 e aceito em março/2016

DOI: $10.15628 /$ holos.2016.3360

\section{RESUMO}

O Tratamento Fora de Domicílio (TFD) é o instrumento legal que visa tornar possível o tratamento, pelo Sistema Único de Saúde (SUS), aos cidadãos portadores de enfermidades não tratáveis em seu município de origem, mediante o custeio de passagens e diárias. Este artigo tem como objetivo geral analisar o processo de implementação do TFD no Piauí e como objetivos específicos: Trazer ao conhecimento a legislação aplicável ao TFD nas esferas federal e estadual bem como promover uma análise acerca da produção de TFD no Piauí. Para tanto se fez a análise da produção de TFD nos anos de 2009 a 2013 em termos de quantidade de procedimentos e dos valores orçamentários e financeiros envolvidos. A abordagem metodológica utilizada tem caráter quanti-qualitativo. A pesquisa apontou para a importância do TFD na busca da universalização do acesso aos serviços de saúde e no processo de regulação. Constatou-se que o TFD foi implementado no Piauí no modelo previsto pela Política Nacional de Regulação. Percebeu-se que no Piauí a maior parte dos pacientes foi tratada no próprio Estado. Os dados da execução orçamentária e financeira revelaram que, no período pesquisado, a fixação dos recursos orçamentários para o TFD ficou aquém dos valores médios da produção aprovada e que a execução financeira representou pouco mais da metade dos recursos orçamentários fixados. Neste sentido, os dados apontaram para um desempenho considerado ineficiente.

PALAVRAS-CHAVE: Use até 05 (cinco) palavras chave, separando-as por vírgula.

\section{THE PROGRAM OF OUT HOME CARE ON SINGLE HEALTHCARE SYSTEM ON PIAUÍ}

\begin{abstract}
The "Out of Home Care" (OHC) is the legal tool which aims to make treatments possible by the "Single Healthcare System" (SHS) for the citizens who bear highly, non-treatable in their original cities through the cost of tickets and daily rates. The research as a study object, and a general goal: to analyze the implementation process of OHC on the state of Piauí, and as specific goals: to know what legislation is to be employed to $\mathrm{OHC}$ on the federal and state spheres and analyzing OHC's production on Piauí. Being so, an analysis of $\mathrm{OHC}^{\prime}$ 's efficiency from the years of 2009 to 2013 has been made, taking in account the amount of procedures and economical values involved. The methodological approach used has as much quantitative characteristics as qualitative ones.
\end{abstract}

The research has highlighted the importance of $\mathrm{OHC}$ in the search for universalizing the access to healthcare services and the regulation. It has also been found that $\mathrm{OHC}$ has been implemented on the state of Piauí according to the model established by the National Regulation Policy. It has been revealing so that most patients have been treated in their own state. The data correspondent to the budgetary and financial execution reveals that during the researched time, the fixation of budgetary resources for $\mathrm{OHC}$ was below the medium valuation for an approved production and that the financial execution represented only little more than half of the fixed budgetary resources. In that sense, the data pointed out to the performance considered inefficient.

KEYWORDS: Use until five (05) keywords by separating them with commas. 


\section{INTRODUÇÃO}

O Programa de Tratamento Fora de Domicílio (TFD) é um instrumento legal que visa garantir, por meio da rede pública de saúde, o atendimento médico a pacientes portadores de doenças não tratáveis em seus municípios de origem por falta de condições técnicas ou profissionais, mediante o custeio das passagens e diárias necessárias para o deslocamento e estada desses pacientes, enquanto durar o tratamento. Dessa forma, constitui-se elo entre o paciente usuário do Sistema Único de Saúde (SUS) e o prestador do serviço de saúde, funciona como instrumento de cidadania e inclusão social, e colabora para o efetivo funcionamento de outras políticas de saúde.

A importância do TFD torna-se mais explícita quando se verifica que muitos usuários do SUS não possuem por vezes condições financeiras para deslocarem-se dos municípios de suas residências, em razão de não encontrarem mais ali, possibilidades para o tratamento adequado do qual necessitam para a conservação ou promoção de sua saúde. Visto dessa forma, não é difícil perceber que o TFD, em muitos casos, pode significar até mesmo a sobrevivência de muitos cidadãos.

Este artigo tem por objetivo divulgar os resultados de uma pesquisa realizada sobre a operacionalização do TFD que se constituiu numa dissertação de mestrado em Políticas Públicas na Universidade Federal do Piauí com o título: "O Programa de Tratamento Fora de Domicílio no Piauí: Uma análise de sua implementação a partir das dimensões jurídica, operacional e financeira nos anos de 2009 a 2013".

Os resultados são apresentados em cinco tópicos, além da introdução. O segundo tópico intitulado de "Políticas de Saúde no Brasil: A Trajetória do Direito à Saúde" aborda a luta para a conquista desse direito. O terceiro tópico tem o título de "A Implementação do Tratamento Fora de Domicílio no Estado do Piauí" apresenta o TFD, seu arcabouço jurídico-legal, o posicionamento dentro da Política Nacional de Regulação (PNR) e suas fontes de financiamento. No quarto tópico, intitulado de "A Produção do Programa de Tratamento Fora de Domicílio no Piauí no período de 2009 a 2013" é feita a apresentação e a análise dos dados coletados sobre o TFD em termos de quantidade e valores de procedimentos e também em termos de execução orçamentária e financeira. No quinto tópico são tecidas as Considerações Finais onde se destaca que o TFD é um importante instrumento na promoção do acesso dos usuários do SUS à rede assistencial de saúde e que no Piauí o mesmo foi implementado de acordo com os ditames da Portaria/SAS no 55/99, cumprindo assim as funções estabelecidas pela Política Nacional de Regulação no sentido de controlar e direcionar o fluxo de pacientes. Com relação ao aspecto quantitativo da produção de TFD aprovada no período analisado destaca-se o bom desempenho do Piauí. Porém, quanto aos aspectos orçamentários e financeiros, a análise aponta para ineficiência tanto da execução orçamentária quanto da execução financeira em relação à produção aprovada.

\section{POLÍTICAS DE SAÚDE NO BRASIL: A TRAJETÓRIA DO DIREITO À SAÚDE}

Os aspectos relacionados com a questão federativa e os serviços de saúde pública sempre estiveram presentes no contexto social e político do Estado brasileiro. 
A assistência à saúde está presente em todas as constituições brasileiras desde o período imperial até a dos dias atuais. Para Augusto (1989), o cuidado com a saúde integra as atribuições governamentais do Estado brasileiro desde que este foi constituído, no entanto a forma como se dá a intervenção estatal e a concepção do que seja promover a saúde pública ou coletiva vem se alterando ao longo do tempo. A questão federalista também presente desde a Proclamação da República em 1889 influencia o desenho das políticas de saúde. De acordo com Viana e Machado (2009), os sistemas de proteção social, por suas características distintas, podem influenciar diferentes arranjos federativos, assim como podem ser influenciados por estes, uma vez que esses sistemas podem o ser elemento chave do padrão de intervenção estatal e do funcionamento da estrutura federativa.

Neto et al. (2010) enfatizam que os problemas de saúde pública da Primeira República eram decorrentes da interdependência sanitária, no sentido de que os problemas atingiam a todas as camadas da sociedade indistintamente, pois as condições de carência e destituição de um determinado grupo social cada vez mais atingia outros grupos. O modelo de proteção social deveria então contemplar todas as classes sociais indistintamente, sendo necessário o envolvimento do governo central e dos governos subnacionais. A tomada de consciência coletiva que levou ao consenso a respeito da necessidade do enfrentamento da interdependência sanitária teve como um dos seus principais atores o Movimento Sanitarista Brasileiro da década de 1910.

O período pós 1930, para as políticas de saúde, pode ser caracterizado como "dual e fragmentado, momento em que dois ministérios federais passam a dividir atribuições na oferta de serviços de saúde" (VIANA; MACHADO, 2009, p.810): o Ministério da Educação e Saúde Pública (MESP) e o Ministério do Trabalho Indústria e Comércio (MTIC), ambos criados em 1930.

Interessava ao governo Vargas, conforme Santos, Barbosa e Gomes (2009), a construção de um Estado nacional, centralizador e intervencionista. $\mathrm{O}$ atendimento às demandas decorrentes dos conflitos urbanos seria implementada pelo MITC enquanto que a da população rural, fora do mercado de trabalho, pelo MESP. No desenho das políticas percebe-se o caráter corporativista das políticas do MTIC e o universalista das políticas do MESP.

O período compreendido dos anos 60 até a promulgação da Constituição de 1988 corresponde ao período da ditadura militar e início do processo de redemocratização do Brasil. Para Viana e Machado (2009), no início dos anos sessenta, discutia-se um novo arranjo federativo, via descentralização com contornos municipalistas. No período autoritário (de 1964 a meados dos anos 80 ) adotou-se um arranjo federativo concentrador, com o poder financeiro e normativo centrado na União.

As características das relações entre os entes governamentais no Estado brasileiro durante a ditadura militar eram, conforme Arretche (2000, p.45), "muito mais próximas às formas que caracterizam um Estado unitário do que daquelas que caracterizam uma federação". Foi nesse cenário que o Sistema Brasileiro de Proteção Social se consolidou, tendo como principais características a centralização financeira e administrativa por parte do governo federal. As políticas de saúde tinham a execução financeira e administrativa centralizada no Instituto Nacional de Assistência Médica da Previdência Social (INAMPS) e nos programas do Ministério da Saúde (de orientação vertical). Aos estados e municípios competia o papel de elaborar seus projetos nos moldes exigidos pelas agências centrais de gestão das políticas de saúde. 
Na década de 80, a realidade social da maior parte dos cidadãos brasileiros era a exclusão do direito à saúde, que na época era prestada pelo INAMPS e se restringia aos trabalhadores com carteira assinada que para ele contribuíam. A atuação do Ministério da Saúde se restringia às atividades de promoção da saúde e prevenção de doenças, as quais eram realizadas em caráter universal, com a assistência médico-hospitalar para poucas doenças e atendimento dos indigentes, considerados como aqueles excluídos do acesso ao atendimento pelo INAMPS. Fernandes (2011) ressalta que a proliferação de serviços privados de saúde incentivados por práticas governamentais para destino de recursos orçamentários para a construção e reforma de hospitais particulares entre os anos de 1970 e 1974 e a crise no financiamento do sistema de previdência social, agravada pela recessão econômica mundial nos anos 80 ajudaram a criar a atmosfera política favorável à discussão de mudanças no sistema de saúde vigente à época.

Nesse mesmo sentido, Silva (2011) enfatiza que a saúde passou a despertar a atenção de outros atores que não os da área técnica do setor, assumindo assim uma dimensão política vinculada à ideia de democracia, este fato contribui para um amplo debate na sociedade civil em torno das condições de saúde da população brasileira. Dos sujeitos políticos coletivos que tiveram participação preponderante nas discussões destacam-se, ainda seguindo o pensamento de Silva (2011), as entidades representativas dos profissionais de saúde, o Movimento Sanitário, os partidos políticos e os movimentos sociais urbanos, estes por meio de eventos em articulação com outras entidades da sociedade civil.

De acordo com informações da página da Biblioteca Virtual Sérgio Arouca, acessada em 14/fev/2015, consta que em 17 de março de 1986 foi aberta a 8a Conferência Nacional de Saúde (8a CNS), evento onde a saúde foi discutida sob três grandes temas: a) Saúde como direito; b) Reformulação do Sistema Nacional de Saúde e; c) Financiamento do setor. Um dos grandes momentos da Conferência foi o consenso obtido em torno do Sistema Único Descentralizado de Saúde (SUDS) que depois se transformaria no SUS.

O movimento da reforma sanitária brasileira e os resultados da 8a CNS levaram ao reconhecimento da saúde como direito de cidadania na Constituição de 1988 e a instituição do SUS. Nesse sentido, instituiu também um novo modelo de assistência à saúde, alicerçado nos princípios da descentralização e regionalização. As mudanças estabelecidas na Constituição viriam impactar profundamente o processo de formulação e implementação das políticas de saúde e as condições de acesso por parte da população aos serviços disponibilizados pela rede pública que se pretendia institucionalizar.

Ao regulamentar o SUS e as ações e serviços de saúde em todo o território brasileiro, a Lei 8080/90 estabeleceu, conforme o artigo 7ำ, a universalidade do acesso aos serviços de saúde em todos os níveis de assistência. Observa-se que o TFD foi instituído como forma de possibilitar que as políticas de saúde possam cumprir as determinações contidas no texto constitucional regulamentado pela Lei 8080/90 de modo universal e igualitário, por meio do encaminhamento de pacientes portadores de moléstias classificadas como de média e alta complexidade aos serviços públicos de saúde, dadas as determinações e limitações do Programa. 


\section{A IMPLEMENTAÇÃO DO TRATAMENTO FORA DE DOMICÍLIO NO ESTADO DO PIAUÍ}

O processo de implementação do TFD no estado do Piauí obedece, dentre outras, às diretrizes gerais da Portaria/SAS no 55 de 24 de fevereiro de 1999 e da Política Nacional de Regulação do Sistema de Saúde (PNR), instituída pela Portaria no 1.559, de 1ㅇ de agosto de 2008 (BRASIL, 2008).

O processo de implementação foi analisado enfatizando-se os aspectos referentes ao arcabouço jurídico que confere a legalidade e legitimidade das ações desenvolvidas pela central de regulação no estado do Piauí além das respectivas fontes de financiamento em nível federal e estadual, em contraposição às quantidades e valores dos procedimentos concedidos pelo TFD no Piauí nos anos de 2009 a 2013.

Procurou-se, dessa forma, analisar a relação entre os valores orçamentários alocados pela Secretaria de Planejamento do Piauí (SEPLAN) no orçamento anual aprovado para os respectivos anos do período pesquisado. A execução financeira efetivada pela Secretaria Estadual de Saúde do Piauí (SESAPI) foi analisada por meio dos dados do Sistema de Administração Orçamentária e Financeira para Estados e Municípios (SIAFEM). Por fim, procurou-se analisar as informações disponibilizadas para acesso público no Departamento de Informática do SUS (DATASUS) referentes aos procedimentos concedidos em termos de quantidades e valores efetivamente pagos. Essa abordagem, leva em consideração o modelo de análise de políticas publicas proposto por Giovanni (2009) e Silva (2013).

Em 24 de fevereiro de 1999, o Ministério da Saúde por intermédio da Secretaria de Assistência à Saúde (SAS) disciplinou a Lei Orgânica da Saúde no 8.080/90 com a edição da Portaria/SAS no 55 que dispõe sobre a rotina do Tratamento Fora de Domicílio no Sistema Único de Saúde - SUS, com inclusão dos procedimentos específicos do programa na Tabela de Procedimentos do Sistema de Informações Ambulatoriais do SIA/SUS além de outras providências com relação à implementação do TFD. Atualmente, os procedimentos referentes ao TFD estão classificados conforme a Tabela 1:

Tabela 1: Procedimentos de TFD na tabela OPM/SUS

\begin{tabular}{|c|c|}
\hline Nomenclatura & Valor(R\$) \\
\hline 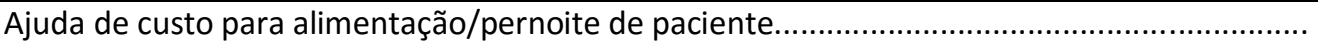 & 24,75 \\
\hline Ajuda de custo $\mathrm{p} /$ alimentação de paciente $\mathrm{s} /$ pernoite.......... & 8,40 \\
\hline Ajuda de custo $p / a$ limentação/pernoite de paciente $(\mathrm{p} /$ tratamento CNRAC) ........................... & 24,75 \\
\hline Ajuda de custo $p / a$ limentação/pernoite de acompanhante....................... & 24,75 \\
\hline 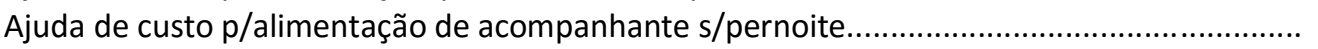 & 8,40 \\
\hline Ajuda de custo $p / a$ limentação/pernoite de acompanhante ( $\mathrm{p} /$ tratamento CNRAC)................ & 24,75 \\
\hline 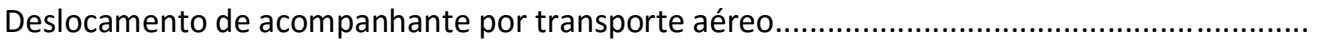 & 181,50 \\
\hline 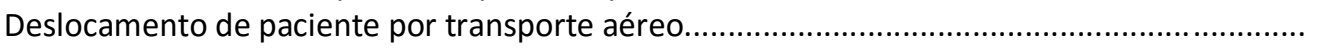 & 181,50 \\
\hline Deslocamento de acompanhante por transporte fluvial.. & 3,70 \\
\hline Deslocamento de acompanhante por transporte terrestre & 4,95 \\
\hline 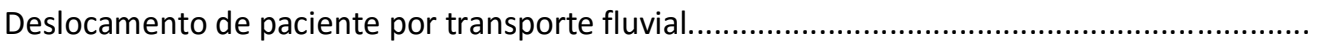 & 3,70 \\
\hline Deslocamento de paciente por transporte terrestre & 4,95 \\
\hline Deslocamento interestadual de acompanhante por transporte aéreo ( $\mathrm{p} /$ tratamento $\mathrm{CNRAC}$ ). & 181,50 \\
\hline Deslocamento interestadual de paciente por transporte aéreo ( $\mathrm{p} /$ tratamento CNRAC)........... & 181,50 \\
\hline
\end{tabular}

Fonte: DATASUS/SIGTAP. Acesso em 02/12/2014. Adaptada pelo autor. 
Percebe-se que a classificação dos procedimentos considera separadamente os procedimentos que devam ser regulados pela Central Nacional de Regulação de Alta Complexidade (CNRAC), assim como classifica os procedimentos referentes ao paciente e ao acompanhante separadamente. Percebe-se também que a legislação garante que, em caso de pernoite, o paciente não fique desacompanhado.

A Política Nacional de Regulação foi instituída em função, dentre outras, da necessidade de estruturação das ações de regulação, controle e avaliação no âmbito do SUS e da necessidade de organizar e garantir o acesso equânime, integral e qualificado aos serviços de saúde da rede assistencial do SUS, objetivando o fortalecimento do processo de regionalização, hierarquização e integração das ações e serviços em saúde. $O$ artigo 20 da PNR estabelece que as ações de regulação são necessariamente integradas entre si e são organizadas em três dimensões: a) Regulação de Sistemas de Saúde; b) Regulação de Atenção à Saúde e; c) Regulação do Acesso à Assistência, dimensão na qual o TFD está inserido.

No Piauí, a estratégia de implementação do TFD foi objeto da pauta da 137ạ Reunião Extraordinária da CIB/PI realizada em 21 de setembro de 2007. A Reunião aprovou por meio da Resolução CIB/PI no 058/2007 a normatização dos benefícios do TFD por intermédio da edição do Manual TFD -SUS /PI, sendo assim, o Manual define em regras gerais as responsabilidades da SES e das SMS para autorização do TFD, os atores, os critérios, rotinas e fluxos operacionais dos recursos financeiros envolvidos no trâmite dos processos de solicitação de TFD, sistematizando dessa forma, as atividades de regulação, autorização e liberação de benefícios no estado do Piauí.

Os recursos orçamentários para o TFD são alocados no orçamento anual do estado pela Secretaria de Planejamento do Estado do Piauí (SEPLAN). As leis orçamentárias que autorizaram as despesas com o TFD no Piauí no período pesquisado conforme dados da SEPLAN, foram as seguintes: a) 2009 - Lei № 5.832, de 30 de dezembro de 2008; b) 2010 - Lei no 5.962, de 07 de janeiro de 2010; c) 2011 - Lei no 6.037, de 30 de dezembro de 2010; d) 2012 - Lei no 6.155, de 05 de janeiro de 2012 e; e) 2013 - Lei no 6.305, de 10 de janeiro de 2013.

Na Tabela 2 estão demonstrados todos os valores autorizados por fonte de recurso e por elemento de despesas discriminadamente.

Tabela 2: TFD Piauí - quadro de detalhamento da despesa

\begin{tabular}{lccccccc}
\hline & \multicolumn{3}{c}{ SUS } & \multicolumn{3}{c}{ TESOURO } & TOTAL \\
\hline & Consumo & Passagens & Diárias & Consumo & Passagens & \multicolumn{1}{c}{ Diárias } \\
QDD 2009 & - & 1.200 .000 & 3.513 .633 & - & 250.000 & 250.000 & 5.213 .633 \\
QDD 2010 & - & 2.000 .000 & 5.070 .450 & - & 500.000 & 500.000 & 8.070 .450 \\
QDD 2011 & - & 3.100 .000 & 4.000 .000 & 100.000 & - & 120.000 & 7.320 .000 \\
QDD 2012 & - & 3.000 .000 & 10.000 .000 & - & 400.000 & 400.000 & 13.800 .000 \\
QDD 2013 & - & 2.000 .000 & 3.998 .849 & - & 400.000 & 400.000 & 6.798 .849 \\
\hline TOTAL & - & 11.300 .000 & 26.582 .932 & 100.000 & 1.550 .000 & 1.670 .000 & 41.202 .932 \\
\hline
\end{tabular}

Fonte: SEPLAN. Acesso em 30/06/2014. Elaborada pelo autor.

A execução orçamentária e financeira dos recursos do governo do Piauí é executada por intermédio do SIAFEM, cuja implantação no Piauí foi instituída pela Lei Ordinária no 5.423 de 20 de dezembro de 2004. A Tabela 3 foi elaborada com base nos dados disponíveis no SIAFEM sobre a execução da despesa do TFD no período pesquisado em termos orçamentários e financeiros. 
Tabela 3: TFD Piauí - execução orçamentária e financeira

\begin{tabular}{lrrrr}
\hline POSIÇÃO EM 31/DEZ & Disponível & Restos a Pagar & Liquidado & Despesa Paga \\
\hline EXEC. DA DESPESA 2009 & $470.343,59$ & $20.136,75$ & $3.921 .268,66$ & $3.798 .178,33$ \\
EXEC. DA DESPESA 2010 & $565.495,37$ & $304.123,08$ & $3.640 .831,55$ & $3.586 .797,06$ \\
EXEC. DA DESPESA 2011 & $1.419 .197,73$ & $265.299,92$ & $5.415 .502,35$ & $5.383 .724,01$ \\
EXEC. DA DESPESA 2012 & $5.783 .150,98$ & $23.627,60$ & $7.213 .221,42$ & $7.142 .248,77$ \\
EXEC. DA DESPESA 2013 & $2.113 .018,76$ & $27.568,99$ & $6.008 .261,25$ & $5.961 .436,81$ \\
\hline
\end{tabular}

Fonte: SIAFEM. Acesso em 30/06/2014. Elaborada pelo autor

Percebe-se no processo de execução orçamentária e financeira do TFD no Piauí, que as ações executadas pelos atores institucionais SEPLAN e SESAPI visam garantir que os objetivos estabelecidos pela PNR em relação à regulação do acesso sejam atingidos, e que as ações desenvolvidas na operacionalização dos procedimentos em termos financeiros sejam executadas de maneira eficiente.

\section{A PRODUÇÃO DO PROGRAMA DE TRATAMENTO FORA DE DOMICÍLIO NO PIAUÍ NO PERÍODO DE 2009 A 2013}

A análise do processo de implementação do TFD no estado do Piauí foi realizada por meio de uma abordagem quanti-qualitativa visando a identificação do volume dos aportes orçamentários e financeiros destinados ao Programa bem como a quantidade e natureza dos benefícios concedidos no período de 2009 a 2013, além dos aspectos do arcabouço jurídico que conferem a legalidade às ações executadas na operacionalização do TFD.

Para atingir esse fim, foram realizados os procedimentos técnicos instrumentais, elaborados conforme a proposta de Silva (2013) sobre os aspectos metodológicos da pesquisa avaliativa, classificados como: a) atividades preliminares ou preparatórias; b) elaboração do plano da pesquisa; c) implementação da pesquisa e; d) o processamento, análise e síntese dos dados e informações.

As informações referentes à análise do processo de implementação do TFD no Piauí foram obtidas com apoio de pesquisa documental, por meio, principalmente, de consulta às leis federais, do estado do Piauí, base de dados do DATASUS e outras fontes documentais oficiais que versam sobre a temática, ancorada em bases conceituais propostas por Giovanni (2009), Silva (2013), Apolinnário (2006) e Spink (2000).

Para melhor visualização dos gráficos que dão suporte às analises da produção do TFD no Piauí, optou-se por reunir os procedimentos em três grupos, segundo a semelhança entre a natureza dos procedimentos. Julgou-se necessário agir dessa forma, pois os dados disponibilizados pelo DATASUS utilizados nessa pesquisa referem-se individualmente aos 14 procedimentos de TFD. Assim, os dados dos procedimentos foram condensados em apenas três classificações denominadas de: a) Ajuda de Custo; b) Passagens Estaduais e; c) Passagens Interestaduais.

O Gráfico 1 demonstra a produção ambulatorial aprovada para os procedimentos do TFD para o estado do Piauí no período de 2009 a 2013. 


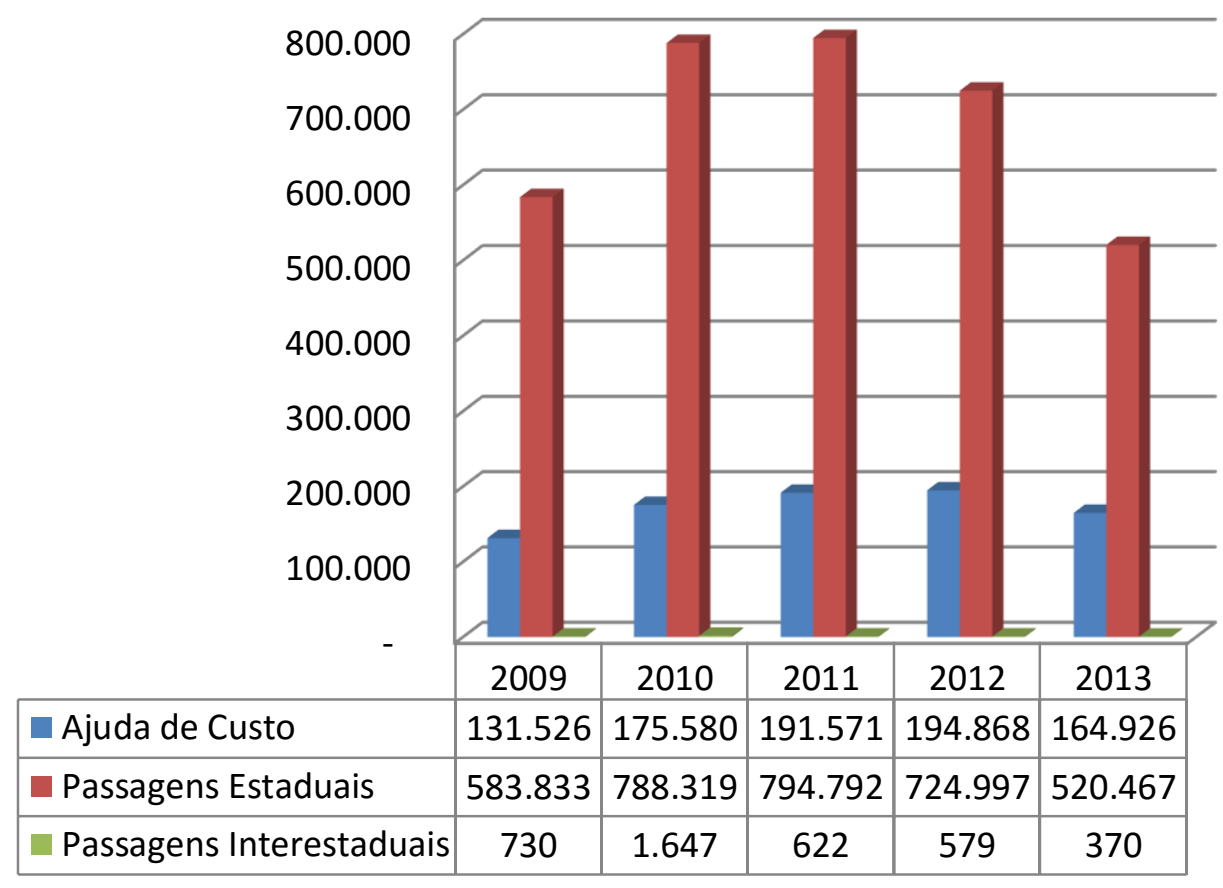

Gráfico 1: TFD Piauí - procedimentos aprovados

Fonte: Ministério da Saúde - SIA/SUS. Acesso em 02/11/2014. Adaptado pelo autor

Os procedimentos referentes a passagens estaduais e ajuda de custo representaram praticamente a totalidade da produção apresentada pelo estado do Piauí com 79,83\% e 20,08\% respectivamente. As passagens aéreas interestaduais representaram apenas $0,09 \%$ do total da produção do período. Esses dados apontam para a constatação de que o tratamento dos pacientes que recorreram aos benefícios do TFD foi realizado quase totalmente dentro do próprio estado, por meio da rede pública ou conveniada/credenciada, sem a necessidade de deslocamentos interestaduais. Essa tendência se manteve constante, com pequenas variações no período observado.

As quantidades totais aprovadas apresentaram uma variação negativa nos anos analisados em termos percentuais no valor de $4,23 \%$. Os procedimentos referentes à ajuda de custo registraram um aumento percentual de $25,39 \%$ no período, no entanto os procedimentos relativos às passagens estaduais e passagens interestaduais apresentaram respectivamente $10,85 \%$ e $49,32 \%$ de variação negativa no período.

A variação negativa da produção é observada principalmente com relação ao ano de 2013, ano que apresentou a menor quantidade durante o período observado. Esse fato, no entanto, não deve ser considerado como negativo em relação à eficiência da operacionalização do TFD, como poderia se supor em uma análise, levando-se em conta apenas a quantidade apresentada no período. Outra suposição plausível poderia ser levantada, considerando que a diminuição de benefícios concedidos pelo programa TFD poderiam indicar um aumento geral na saúde pública ou maior resolutividade dos entes municipais, porém seriam necessários dados adicionais sobre a rede estadual de saúde do Piauí. O Gráfico 2 demonstra a produção aprovada no período pesquisado em termos de valores monetários: 


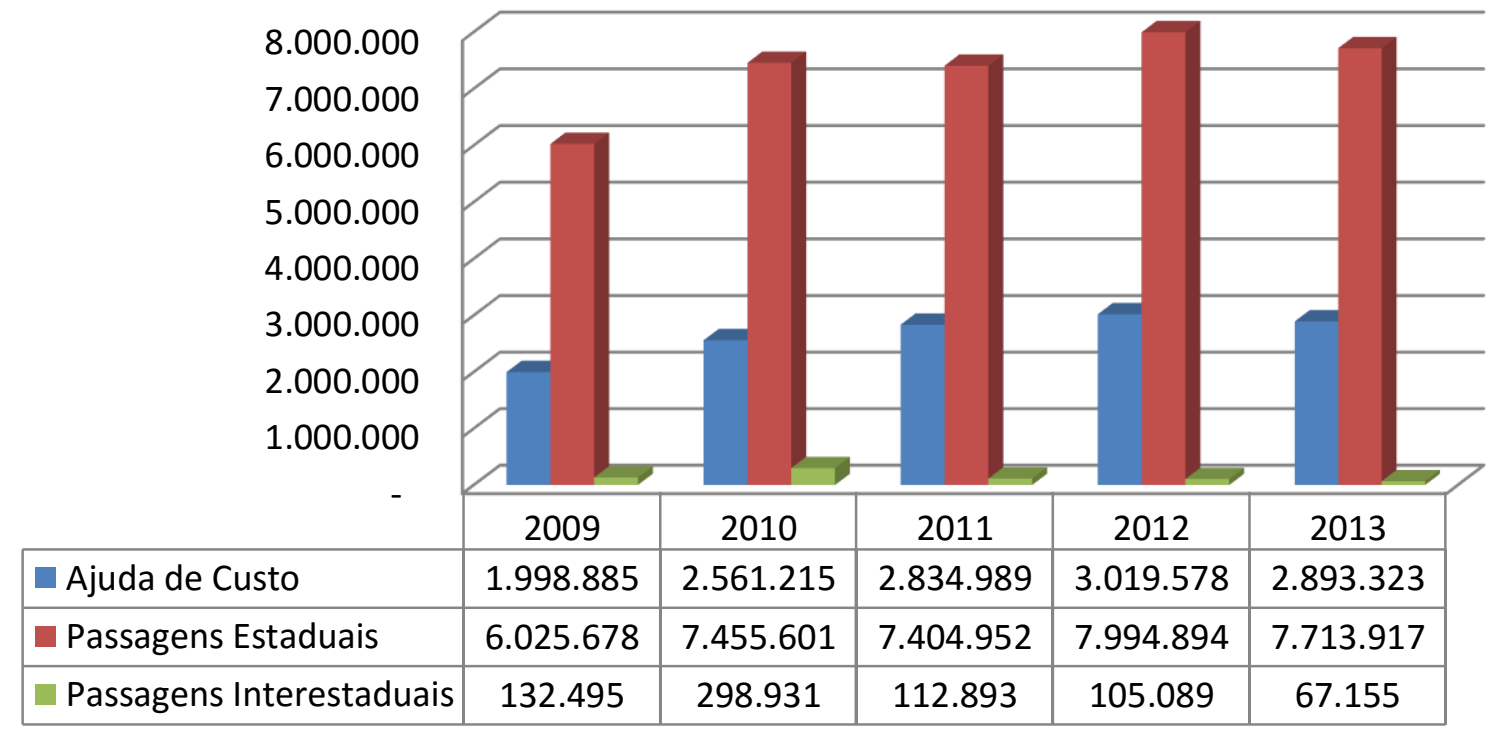

Gráfico 2 : TFD Piauí - valores aprovados

Fonte: Ministério da Saúde - SIA/SUS. Acesso em 02/11/2014. Adaptado pelo autor

Mediante análise do Gráfico 2, percebeu-se que no Piauí houve aumento na produção aprovada no período pesquisado em termos de valores originais na ordem de $30,86 \%$. Contudo, esse aumento não foi constante para todos os procedimentos. O aumento apresentado pelo item "Ajuda de Custo" representou uma variação positiva de 44,75\%; o mesmo comportamento foi observado no item "Passagens Estaduais", com variação positiva de 28,82\%, porém, o item "Passagens Interestaduais" apresentou variação negativa de 49,32\%. A constatação da redução de praticamente metade dos procedimentos de TFD de natureza interestadual e o consequente aumento dos procedimentos de natureza intermunicipal levam inicialmente à suposição de uma possível ampliação no atendimento e de melhoria na resolutividade da rede assistencial do Piauí, ou que talvez sejam resultantes de mudanças socioeconômicas havidas na região, uma vez que os valores são tabelados e não sofreram alteração no período pesquisado. No entanto, pode-se supor também que o aumento dos procedimentos intermunicipais se dá em função da escassez de serviços de saúde nos municípios do Piauí.

O Gráfico 3 demonstra a relação entre os valores da produção de TFD aprovada para Piauí e os valores efetivamente pagos pela SESAPI no período de 2009 a 2013:

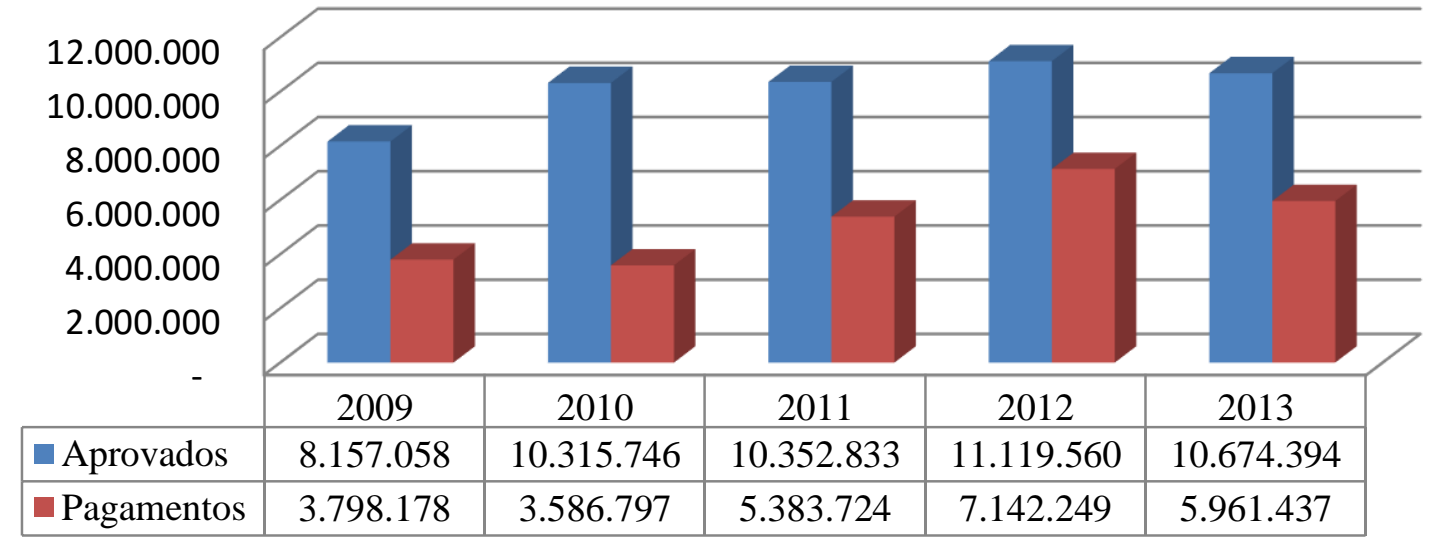

Gráfico 3: TFD Piauí - valores aprovados $X$ pagamentos

Fonte: SIAFEM. Acesso em 30/06/2014. Adaptado pelo autor 
Os valores orçamentários alocados para o TFD no período de 2009 a 2013, de maneira geral ficaram abaixo dos valores da produção aprovada, o que implica dizer que os valores pagos também tiveram comportamento idêntico. Essa constatação trouxe à tona pelo menos dois aspectos a respeito da implementação do TFD no Piauí e que implicam, em última análise, na garantia do acesso dos usuários às ações e serviços de saúde disponibilizados pela rede assistencial do SUS.

A primeira, com relação à previsão orçamentária: A constatação pelos dados do período analisado de que a previsão orçamentária dos recursos foi insuficiente para fazer face aos pagamentos dos benefícios aprovados pelo TFD no Piauí. A segunda constatação, com relação aos pagamentos efetivamente autorizados para o TFD: A execução financeira do TFD em patamares abaixo da metade dos valores aprovados demonstrou a dificuldade do governo estadual em converter os valores fixados pelo orçamento em disponibilidade financeira, alocada ao TFD, para pagamento dos processos autorizados, por meio da contrapartida estadual. Essa dificuldade implica pelo menos em duas questões: A capacidade de arrecadação de tributos de competência do Estado e a efetiva alocação dos recursos arrecadados, com o intuito de pagamento da contrapartida de responsabilidade do estado. O enfrentamento dessas possíveis dificuldades implica em decisões administrativas no sentido da adoção de critérios mais consistentes quando da elaboração do orçamento, do monitoramento da execução orçamentária e dos pagamentos por meio da instituição de rotinas de auditoria interna nas ações e setores envolvidos com os processos de TFD. Cabe ressaltar que essas observações levaram em consideração que o SUS repassa os valores de responsabilidade da União por meio dos pagamentos da produção ambulatorial de média e alta complexidade.

\section{CONSIDERAÇÕES FINAIS}

O acesso universal e igualitário estabelecido como princípio do SUS vem sendo estabelecido de forma gradual em função da complexidade decorrente das enormes diferenças regionais e da pluralidade de contextos vivenciados pelos municípios brasileiros.

O acesso à saúde engloba também fatores de ordem política, cultural, econômica e funcional que podem resultar em dificuldades que impedem, por um lado a satisfação das necessidades de assistência de grande parte da população. Esses fatores, por outro lado, dificultam a disponibilização por parte do governo de uma estrutura de saúde que garanta serviços de boa qualidade capazes de atender ao cidadão em seu município de origem

Diante da necessidade de organizar e controlar o fluxo de pacientes que recorrem à rede assistencial pública de saúde instituiu-se a Política Nacional de Regulação do SUS, como forma de possibilitar aos três níveis de governo a execução de ações direcionadas a tornar universal o acesso aos serviços públicos de saúde, por meio da regulação do acesso à assistência, dimensão na qual o TFD está inserido.

O TFD, ao atingir de forma eficiente o seu objetivo, que é o de proporcionar o acesso dos usuários do SUS aos serviços necessários para o restabelecimento de sua saúde, na forma de passagens e ajuda de custo para hospedagem e alimentação enquanto durar o tratamento, tornase elo entre o paciente e o tratamento e contribui de maneira definitiva para tornar possível o princípio da universalização do acesso à assistência à saúde. Constitui-se também instrumento 
inconteste para a consecução dos objetivos da Política Nacional de Regulação, pois viabiliza o acesso do tratamento de saúde aos usuários do SUS e contribui para mitigar os efeitos decorrentes da falta de estrutura da rede assistencial, principalmente nos municípios das regiões mais distantes do Brasil.

A análise desenvolvida nessa pesquisa permite constatar a implementação do TFD no Piauí em pelo menos dois aspectos: No primeiro, a implementação do ponto de vista da observação da fundamentação legal e operacionalização do fluxo do processo de pedido de TFD: Percebe-se que as disposições da legislação aplicável ao TFD foram obedecidas satisfatoriamente em termos de operacionalização das ações de aprovação dos processos, o que reflete o volume de procedimentos aprovados, demonstrando que no Piauí, o acesso dos usuários do SUS à rede assistencial pode ser considerado satisfatório. No segundo aspecto, a implementação do ponto de vista da execução orçamentária e financeira, o desempenho do Piauí demonstra ineficiência em função dos baixos índices apresentados. No entanto, essa ineficiência não pode ser explicada somente a partir dos dados pesquisados, neles não se podem perceber as causas da ineficiência, apenas seus efeitos refletidos na execução orçamentária e financeira dos recursos alocados para o TFD.

\section{REFERÊNCIAS}

1. ARRETCHE, M.T. S. Estado federativo e políticas sociais: determinantes da descentralização. Rio de Janeiro: Revan; São Paulo: FAPESP, 2000.

2. AUGUSTO, M.H.O. Políticas públicas, políticas sociais e política de saúde: algumasquestões para reflexão e debate. Tempo Social; Rev. Sociol. USP, S. Paulo, v.1, n. 2, p. 105-119, 2 .sem 1989.

3. APPOLINÁRIO, Fábio. As dimensões da pesquisa. In: Metodologia Científica - Filosofia e prática da pesquisa. São Paulo: Thompson Learning, 2006, p.59-72.

4. BRASIL. Ministério da Saúde. Lei 8080 de 19 de setembro de 1990. Disponível em <https://www.planalto.gov.br/ccivil_03/leis//8080.htm> Acesso em 14 set.2014.

5. BRASIL. Ministério da Saúde. Secretaria de Assistência à Saúde. Portaria/SAS/No55 de 24 de fevereiro 1999. Disponível em <http://drt2001.saude.gov.br/sas/PORTARIAS/Port99/PT055.html> Acesso em 04 out. 2014.

6. Ministério da Saúde. Gabinete do Ministro. Portaria/GM/№1559 de 01 de agosto de 2008. Disponível em: <http://bvsms.saude.gov.br/bvs/saudelegis/gm/2008/prt1559_01_08_ 2008.html> Acesso em 01 nov. 2014.

7. FERNANDES, A. J. C. Origens do movimento sanitário brasileiro. Universidade de Brasília, 2011. Disponível em: <http://bdm.unb.br/bitstream/10483/2682/1/2011_AnaJuliaCastroFernandes .pdf> Acesso em 05 dez. 2014.

8. GIOVANNI, G. de. As estruturas elementares das políticas públicas. Caderno de pesquisa. n. 82, Campinas, 2009.

9. NETO, M. V. F. et al. O processo histórico de construção do Sistema Único de Saúde brasileiro e as novas perspectivas in Revista Âmbito Jurídico, no 76 - Ano XIII - Maio/2010. Disponível em < http://www.ambito-juridico.com.br/site/index.php?n_link=revista_artigos_leitura\&arti 
go_id=7781\&revista_caderno=27 > Acesso em 05 dez. 2014.

10. SANTOS, L. A. C.; BARBOSA, I. C.; GOMES, Mauro de Lima. Saúde no governo Vargas (19301945): dualidade institucional de um bem público. Cad. Saúde Pública, Rio de Janeiro , v. 25, n. 9, Set. 2009 . disponível em: <http://www.scielo.br/scielo.php?script=sci_art text\&pid=S0102-311X2009000900023\&Ing=en\&nrm=iso>. Acesso em 28 nov. 2014.

11. SEPLAN. Secretaria do Planejamento. Governo do Estado do Piauí. Orçamento 2009. Disponível em <http://www.seplan.pi.gov.br/upe/Orçamento/orçamento_2009/qdd2009.pdf> página116 Acesso em 29 abr. 2014.

12.

Secretaria do Planejamento. Governo do Estado do Piauí. Orçamento 2010. Disponível em <http://www.seplan.pi.gov.br/upe/Orçamento/orçamento_2010/7-qddexecutivo.pdf>página100 Acesso em 29 abr. 2014.

13. . Secretaria do Planejamento. Governo do Estado do Piauí. Orçamento 2011. Disponível em <http://www.seplan.pi.gov.br/upe/Orçamento/orçamento_2011/lei/QDD_ EXECUTIVO_FIN AL.pdf > página103 Acesso em 29 abr. 2014.

14. Secretaria do Planejamento. Governo do Estado do Piauí. Orçamento 2012. Disponível em <http://www.seplan.pi.gov.br/upe/Orçamento/orçamento_2012/QDD_2012_ EXECUTIVO_FINAL.pdf>página100 Acesso em 29 abr. 2014.

15. SEPLAN. Secretaria do Planejamento. Governo do Estado do Piauí. Orçamento 2013. Disponível em <http://www.seplan.pi.gov.br/upe/Orçamento/orçamento_2014/QDD_Execu tivo/17000.pdf> página 6 Acesso em 29 abr. 2014.

16. SILVA, M. O. S. Avaliação de políticas e programas sociais: uma reflexão sobre o conteúdo teórico e metodológico da pesquisa avaliativa. In: (coord.). Pesquisa Avaliativa: aspectos teórico e metodológicos. São Paulo: Veras, 2013.

17. SILVA, A. X. A reforma sanitária brasileira em debate. In. Jornada Internacional de Políticas Públicas, v, 2011, São Luís. Anais...São Luís. 2011. Disponível em <http://www.joinpp.uf ma.br/jornadas/joinpp2011/CdVjornada/vjornada.html> Acesso em 5 dez. 2014.

18. SPINK, P. Análise de documento de domínio público. In: SPINK, M.J. (org.) Práticas discursivas e produção de sentido quotidiano. São Paulo: Cortez Editora, 2000, pp 123-151.

19. TFD. Tratamento Fora do Domicílio. Disponível em <http://www.mp.pi.gov.br/saude/legisla ção-e-outros/category/660-tratamento-fora-de-domicílio> Acesso em 06 out. 2012.

20. VIANA, A. L. A; MACHADO, C. V. Descentralização e coordenação federativa: a experiência brasileira na saúde. Ciênc. saúde coletiva, Rio de Janeiro , v. 14, n. 3, jun. 2009. Disponível em:<http://www.scielo.br/scielo.php?script=sci_arttext\&pid=S1413-81232009000300016\& Ing=pt\&nrm=iso $>$. Acesso em 03 dez. 2014. 